\title{
Particle identification using the time-over-threshold method in the ATLAS Transition Radiation Tracker
}

T. Akesson ${ }^{\mathrm{a}}$, E. Arik ${ }^{\mathrm{b}}$, K. Assamagan ${ }^{\mathrm{c}}, \mathrm{K}$. Baker $^{\mathrm{c}}$, E. Barberio ${ }^{\mathrm{d}}$, D. Barberis ${ }^{\mathrm{d}, 1}$, H. Bertelsen ${ }^{\text {e }}$, V. Bytchkov ${ }^{\mathrm{f}}$, J. Callahan ${ }^{\mathrm{g}}$, A. Catinaccio ${ }^{\mathrm{d}}$, H. Danielsson ${ }^{\mathrm{d}}$, F. Dittus ${ }^{\mathrm{d}}$, B. Dolgoshein ${ }^{\mathrm{h}}$, N. Dressnandt ${ }^{\mathrm{i}}$, W.L. Ebenstein ${ }^{\mathrm{j}}$, P. Eerola ${ }^{\mathrm{a}}$,

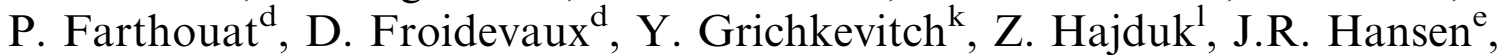
P.T. Keener ${ }^{\mathrm{i}}$, G. Kekelidze ${ }^{\mathrm{f}}$, S. Konovalov ${ }^{\mathrm{m}}$, T. Kowalskin ${ }^{\mathrm{n}}$, V.A. Kramarenko ${ }^{\mathrm{k}}$,

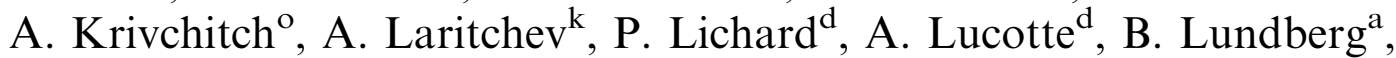
F. Luehring ${ }^{\mathrm{g}}$, A. Mailov ${ }^{\mathrm{b}}$, A. Manara ${ }^{\mathrm{g}}$, K. McFarlane ${ }^{\mathrm{c}}$, V.A. Mitsou ${ }^{\mathrm{d}, 2}$, S. Morozov ${ }^{\text {h }}$, S. Muraviev ${ }^{\mathrm{m}}$, A. Nadtochy ${ }^{\mathrm{o}}$, F.M. Newcomer ${ }^{\mathrm{i}}$, J. Olszowskal, H. Ogren ${ }^{\text {g,* }}$, S.H. Oh ${ }^{\mathrm{j}}$, V. Peshekhonov ${ }^{\mathrm{f}}$, C. Rembser ${ }^{\mathrm{d}}$, A. Romaniouk ${ }^{\mathrm{h}, \mathrm{d}}$, D. Rousseau ${ }^{\mathrm{d}, 3}$, D.R. Rust ${ }^{\mathrm{g}}$, V. Schegelsky ${ }^{\mathrm{o}}$, M. Sapinski ${ }^{1}$, A. Shmeleva ${ }^{\mathrm{m}}$, S. Smirnov ${ }^{\text {, L.N. Smirnova }}{ }^{\mathrm{k}}$, V. Sosnovtsev ${ }^{\mathrm{h}}$, S. Soutchkov ${ }^{\mathrm{h}}$, E. Spiridenkov ${ }^{\mathrm{o}}$, V. Tikhomirov ${ }^{\mathrm{m}}$, R. Van Bergi, V. Vassilakopoulos ${ }^{\mathrm{j}}$, C. Wang ${ }^{\mathrm{j}}$, H.H. Williams ${ }^{\mathrm{i}}$

\author{
${ }^{a}$ Fysiska Institutionen, Lunds Universitet, Lund, Sweden \\ ${ }^{\mathrm{b}}$ Department of Physics, Bogazici University, Istanbul, Turkey \\ ${ }^{\mathrm{c}}$ Hampton University, Hampton, VA 23668, USA \\ ${ }^{\mathrm{d}}$ European Laboratory for Particle Physics (CERN), Geneva, Switzerland \\ ${ }^{\mathrm{e}}$ Nils Bohr Institute, University of Copenhagen, Copenhagen, Denmark \\ ${ }^{\mathrm{f}}$ Joint Institute of Nuclear Research, Dubna, Russia \\ ${ }^{\mathrm{g}}$ Department of Physics, Indiana University, rm. 263, Swain west, Bloomington, IN 47405, USA \\ ${ }^{\mathrm{h}}$ Moscow Engineering and Physics Institute, Moscow, Russia \\ ${ }^{i}$ Department of Physics and Astronomy, University of Pennsylvania, Philadelphia, PA, USA \\ ${ }^{\mathrm{j}}$ Physics Department, Duke University, Durham, NC, USA \\ ${ }^{\mathrm{k}}$ Moscow State University, Institute of Nuclear Physics, Moscow, Russia \\ ${ }^{1}$ Henryk Niewodniczanski Institute of Nuclear Physics, Cracow, Poland \\ ${ }^{\mathrm{m}}$ P.N. Lebedev Institute of Physics, Moscow, Russia \\ ${ }^{\mathrm{n}}$ Faculty of Physics and Nuclear Techniques of the Academy of Mining and Metallurgy, Cracow, Poland \\ ${ }^{\circ}$ Petersburg Nuclear Physics Institute, Gatchina, St. Petersburg, Russia
}

\footnotetext{
*Corresponding author. Tel.: + 1-812-855-2992; fax: + 1-812-855-0440

E-mail address: ogren@indiana.edu (H. Ogren).

${ }^{1}$ Also at University of Genova, Genova, Italy.

${ }^{2}$ Also at University of Athens, Physics Department, Athens, Greece.

${ }^{3}$ Now at Laboratoire de l'Accélérateur Linéaire, Orsay, France.
} 


\begin{abstract}
Test-beam studies of the ATLAS Transition Radiation Tracker (TRT) straw tube performance in terms of electronpion separation using a time-over-threshold method are described. The test-beam data are compared with Monte Carlo simulations of charged particles passing through the straw tubes of the TRT. For energies below $10 \mathrm{GeV}$, the time-overthreshold method combined with the standard transition-radiation cluster-counting technique significantly improves the electron-pion separation in the TRT. The use of the time-over-threshold information also provides some kaon-pion separation, thereby significantly enhancing the B-physics capabilities of the ATLAS detector. (C) 2001 Elsevier Science B.V. All rights reserved.
\end{abstract}

Keywords: Time-over-threshold; Straw; Particle identification

\section{Introduction}

The Transition Radiation Tracker (TRT) [1] is a part of the Inner Detector of the ATLAS experiment. It is designed to provide powerful pattern recognition capabilities and electron identification via transition radiation.

The TRT is a straw drift-tube detector, with a central barrel section and two end-cap sides, giving an average of 35 crossed straws per track. In the barrel TRT, axial straws are embedded in fibre radiators, while, in the end-caps, layers of radial straws alternate with foil radiators. These geometries have been chosen in order to have an approximately constant number of straws crossed by charged particles with $p_{\mathrm{T}}>0.5 \mathrm{GeV}$ over the full rapidity coverage $(|\eta|<2.5)$. Transition radiation is produced when a charged ultra-relativistic particle crosses the interface between different media, mainly polypropylene (fibres or foils) and air in the case of the TRT. The transition-radiation photons are emitted at very small angle with respect to the parent-particle trajectory and are therefore most often detected in the same straws as those crossed by the parent particle.

The TRT straw drift-tubes provide two types of information: the distance of closest approach of the track to the anode wire (for tracking purposes via drift-time measurements) and the energy deposited in the straw (for particle identification). This energy deposition is the sum of the ionisation losses of the charged particle crossing the straw and of the usually larger energy depositions due to transition-radiation photon absorption.
The low-threshold discriminator level is set to a nominal value of $200 \mathrm{eV}$, which is safely above the noise level $(\sigma \sim 40 \mathrm{eV})$ and significantly below the average energy loss of $\sim 2 \mathrm{keV}$, expected for minimum-ionising particles traversing the straw gas. With this low-threshold setting, the front-end electronics detects the initial ionisation cluster for precise drift-time determination.

The high-threshold discriminator level is set to a nominal value of $5 \mathrm{keV}$, which is optimised for $\mathrm{e} / \pi$ separation. Particle identification studies based on the cluster-counting technique with different TRT prototypes have been reported in [2-4]. In these studies, e/ $\pi$ separation was achieved by counting the number of high-threshold hits along the reconstructed track. For electrons, the tail above $5 \mathrm{keV}$ is dominated by transition-radiation hits, while for pions it is mostly due to $\delta$-rays. By requiring more than a certain number of highthreshold hits along the track, the probability to misidentify pions as electrons was measured as a function of the electron efficiency. This procedure is illustrated in Fig. 1a, where a display of a simulated $\mathrm{B}_{\mathrm{d}}^{0} \rightarrow \mathrm{J} / \Psi \mathrm{K}_{\mathrm{s}}^{0}$ event (at the low luminosity nominal value of $\left.10^{33} \mathrm{~cm}^{-2} \mathrm{~s}^{-1}\right)$ in the ATLAS barrel TRT is shown, and in Fig. 1b, where portions of a pion track from the $\mathrm{K}_{\mathrm{s}}^{0}$ decay and of an electron track from a $\mathrm{J} / \Psi$ decay are shown in an enlarged frame (see box in Fig. 1a). Dots represent straws crossed by charged particles. Electron tracks contain many more high-threshold hits (larger points) than pion tracks.

Particle identification using only the information from the low-threshold discriminator has also been shown to be possible [5-7]. A time-over- 

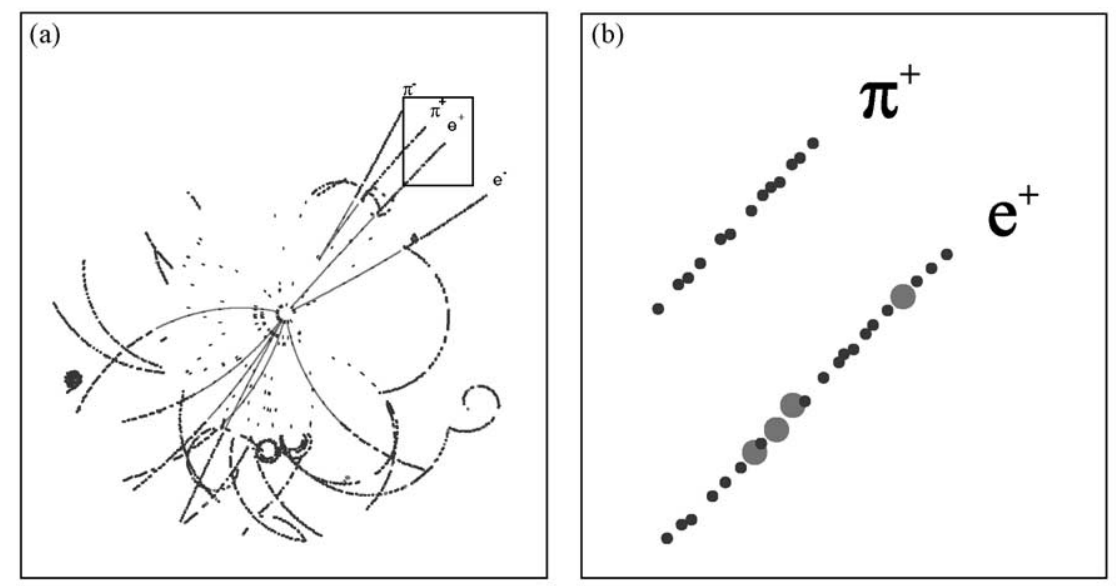

Fig. 1. The display of a simulated $\mathrm{B}_{\mathrm{d}}^{0} \rightarrow \mathrm{J} / \Psi \mathrm{K}_{\mathrm{s}}^{0}$ event in the ATLAS barrel Inner Detector, at low luminosity $\left(10^{33} \mathrm{~cm}^{-2} \mathrm{~s}^{-1}\right)$ is shown in Fig. 1a. The small box selects a part of a pion track from the $\mathrm{K}_{\mathrm{s}}^{0}$ decay and of an electron track from a $\mathrm{J} / \Psi$ decay, shown in an enlarged frame in Fig. 1b.

threshold technique for straw tubes, similar to the one discussed in this paper, has been studied in Ref. [5], whereas the discriminator leading-edge information corrected as a function of track distance from the wire was used in Ref. [6].

In this paper, a time-over-threshold method which includes corrections for the track position inside the straw is discussed. The time-overthreshold is defined as the width of the lowthreshold discriminator signal. This signal width, larger for signal with larger amplitudes, depends on the particle's energy loss and, for electrons, also on transition radiation. However, this width also depends on the track position inside the straw, as illustrated in Fig. 2. For a track crossing the straw near the anode wire, the signal width is obviously larger than for the case when the track crosses the straw near the cathode. In order to extract information about the energy loss of a particle, it is important to correct for this dependence. This results in a significant improvement of the TRT particle identification capability with respect to the method without track-position correction used in Ref. [5], as shown in Section 2.2.2. The time-overthreshold method with track-position correction improves particle identification in the TRT at low particle momenta. The average energy losses expected for electrons, pions and kaons as they travel through the TRT Xe-based gas mixture, are

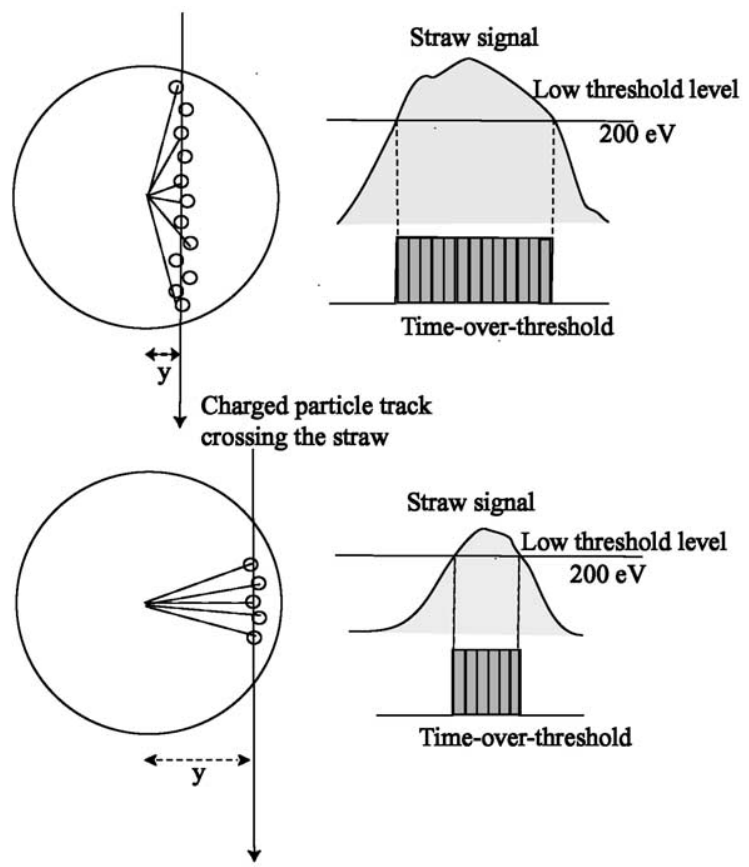

Fig. 2. Dependence of the time-over-threshold on the track distance, $y$, from the wire. The straw signal is shown before and after the low-threshold $(200 \mathrm{eV})$ discrimination.

shown as a function of momentum in Fig. 3. These curves suggest that improved $\mathrm{e} / \pi$ separation may be possible for momenta below $10 \mathrm{GeV}$ and that $\mathrm{K} / \pi$ separation may be possible over the momen- 


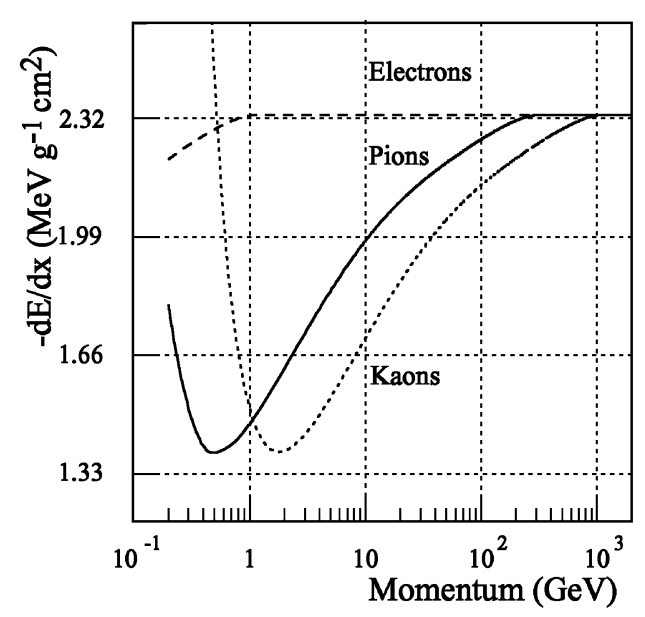

Fig. 3. Bethe-Bloch curves for various particles in the ATLAS TRT gas mixture. For momenta less than $20 \mathrm{GeV}$, good particle identification based on an accurate measurement of the energy loss is possible.

tum range relevant for B-physics at the LHC (see Section 3).

\section{Electron-pion separation}

\subsection{Experimental set-up}

The performance of the time-over-threshold method for electron-pion separation was evaluated in the $\mathrm{H} 8$ beam line at the CERN SPS. Pion and electron beams of different energies were used (20, 10 and $5 \mathrm{GeV})$.

The experimental set-up is shown in Fig. 4. It included a small straw prototype used for precise ionisation loss and transition-radiation measurements ('ADC straws'), and a single straw used for time-over-threshold measurements ('ToT straw'). Other equipment consisted of two standard multiwire beam chambers ( $\mathrm{BC} 1$ and $\mathrm{BC} 2)$ for a first coarse track reconstruction, two silicon detectors (Si1 and Si2) with an intrinsic accuracy of about $10 \mu \mathrm{m}$, which provided a very precise reconstruction of the beam track position in the TRT straws, and two small scintillators (s1 and s2) used in coincidence as the trigger. Two Cherenkov detectors and a lead-glass calorimeter were also read out and used for particle identification.
The straw prototype, placed between the two silicon detectors, had 10 layers of straw drift-tubes, optionally interleaved with radiator (the presence and type of the radiator was run-dependent), and was read out with conventional ADCs. The information from these drift-tubes was used to tune the Monte Carlo model and to study the transition-radiation performance of different radiators inserted between them. The single straw used for the time-over-threshold method was placed behind the second silicon detector, and was rotated by $21^{\circ}$ from the perpendicular to the beam. A $12 \mathrm{~cm}$ thick fibre radiator was positioned in front of this straw, in order to simulate a straw at $|\eta|=0.3$ in the ATLAS barrel TRT (see Section 2.3.2). This single straw was equipped with an amplifier-shaper-discriminator (ASDBLR), with low-level $(200 \mathrm{eV})$ and high-level $(5 \mathrm{keV})$ discriminator thresholds. For this study, the discriminated signal was fed into a multi-hit TDC, with a $1 \mathrm{~ns}$ resolution, that provided leading and trailing edge information both for low and high-threshold signals. In the final version of the ATLAS TRT front-end electronics, this information is extracted using an amplifier-shaper-discriminator (ASDBLR) and a time digitiser (DTMROC). In the ASDBLR the signal is amplified, shaped and two-fold discriminated. In the DTMROC the lowthreshold discriminated pulse is digitised in 3.125 ns time bins. This bin size has been chosen as an acceptable compromise between the measurement accuracy and data bandwidth requirements [8]. From the DTMROC readout the time of the leading and trailing edge of the low-threshold discriminator signal and the possible presence of the high-threshold discrimination level are determined.

Standard TRT $4 \mathrm{~mm}$ diameter Kapton straws with a $30 \mu \mathrm{m}$ diameter gold-plated tungsten wire were used. The straws were $10 \mathrm{~cm}$ in length. The ionisation gas mixture was $69.5 \% \mathrm{Xe}+22 \% \mathrm{CF}_{4}+$ $8.5 \% \mathrm{CO}_{2}$, very close to the one which will be used for the ATLAS TRT $\left(70 \% \mathrm{Xe}+20 \% \mathrm{CF}_{4}+\right.$ $10 \% \mathrm{CO}_{2}$ ). This gas mixture provides good absorption for photons in the X-ray region, a short total drift time of about $40 \mathrm{~ns}$ and good operational stability with respect to discharges. The gain was set to $2.5 \times 10^{4}$ and stabilised through a 


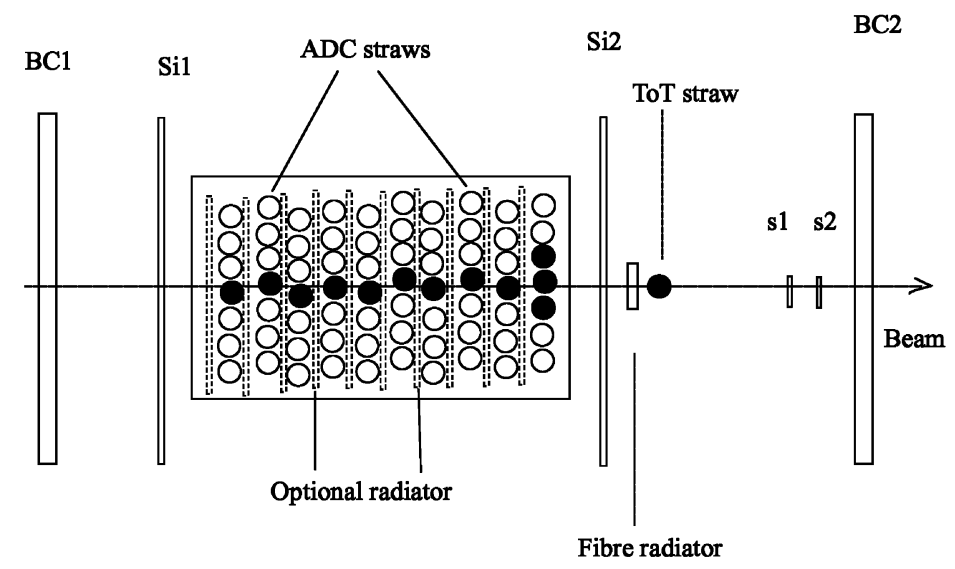

Fig. 4. Schematic view of the experimental set-up (not to scale). MWPCs (BC1 and BC2) and silicon detectors ( $\mathrm{Si} 1$ and $\mathrm{Si} 2)$ were used for tracking, two scintillators (s1 and s2) were used in coincidence for triggering. Straws for radiator studies (ADC straws), the single straw for the time-over-threshold studies (ToT straw) and the radiator locations are also indicated. The straws that were read out are represented as black circles.

high-voltage feedback loop with an accuracy of better than $1 \%$, as nominally required for operation in ATLAS .

\subsection{Data analysis}

\subsubsection{Time-over-threshold studies with a single straw}

The results of the data analysis for the 'ToT straw' in the experimental set-up with all radiators in place (see Fig. 4) are discussed in this section. For this configuration, the amount of transition radiation generated by the 5,10 and $20 \mathrm{GeV}$ electrons corresponded to a probability of about $26 \%$ per straw for high-threshold hits, with energy above $5 \mathrm{keV}$, close to that expected in the ATLAS barrel TRT (see Section 2.3.2).

With incident pions, the high-threshold hit probability was about $6 \%$, mostly due to large energy depositions caused by $\delta$-rays, again close to that expected in the ATLAS barrel TRT.

Since there was no magnetic field, the track reconstruction was performed using a simple linear fit. A coarse track was reconstructed using hits from $\mathrm{BC} 1$ and $\mathrm{BC} 2$, and the track fit was then refined using the very accurate information from the silicon detectors. The straw was aligned by minimising the residual between the predicted position of the track within the straw and the position calculated using the drift-time information. A spatial resolution ranging between $125 \mu \mathrm{m}$ at $20 \mathrm{GeV}$ and $155 \mu \mathrm{m}$ at $5 \mathrm{GeV}$ was achieved. The degradation at lower energy is due to multiple scattering in the material of the set-up.

The time-over-threshold method was evaluated in a way compatible with the ATLAS TRT electronics read out, by emulating the $3.125 \mathrm{~ns}$ DTMROC binning using the data obtained from the 1 ns TDC.

The uncorrected time-over-threshold data obtained in this way for the ToT straw and for $5 \mathrm{GeV}$ pions (solid line) and electrons (dashed line) are shown in Figs. 5 and 6. In Fig. 5, only hits without high-threshold discriminator level are included, since they provide a sample of hits used subsequently to build up a discriminating variable totally independent of the standard transitionradiation cluster-counting technique. In Fig. 6, all the hits are considered, and the difference between pions and electrons is, as expected, enhanced with respect to Fig. 5. The larger values in the timeover-threshold distributions of Fig. 6 are dominated by transition radiation for electrons and by $\delta$-rays for pions.

The correlation between the uncorrected time-over-threshold and the distance of closest 


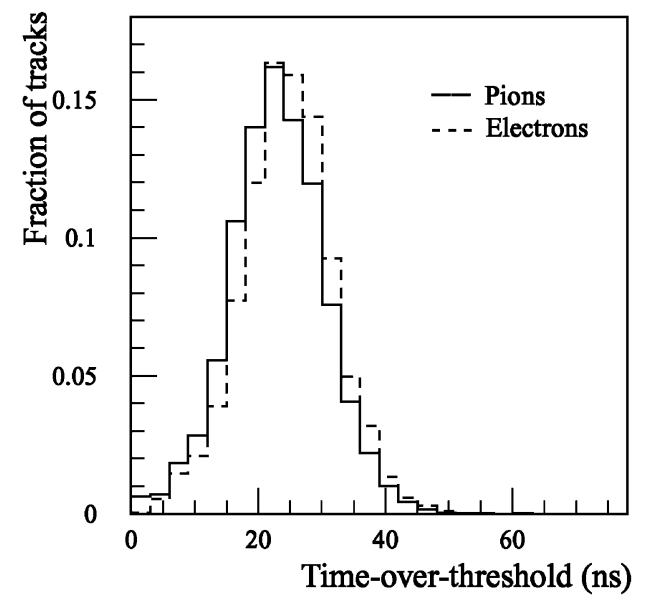

Fig. 5. Uncorrected time-over-threshold distributions for $5 \mathrm{GeV}$ pions and electrons for hits without high-threshold discriminator level.

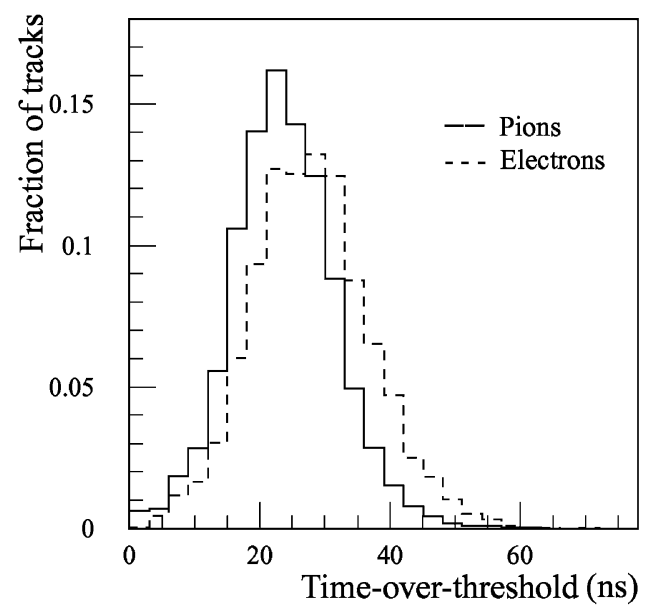

Fig. 6. Uncorrected time-over-threshold distributions for $5 \mathrm{GeV}$ pions and electrons for all hits.

approach to the wire for $5 \mathrm{GeV}$ pion data is shown in Fig. 7 for the sample without high-threshold hits. For a track crossing the straw close to the anode wire, the electron clusters are produced along a length equal to the straw diameter, as sketched in Fig. 2. In order to parameterise this dependence, the straw was divided into $200 \mu \mathrm{m}$ bins and the time-over-threshold was evaluated for each bin. The parameterisation used a cubic polynomial.

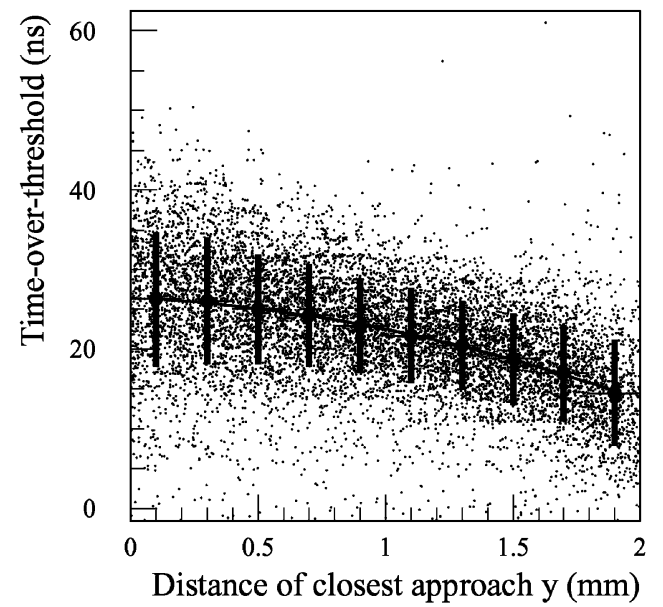

Fig. 7. Parameterisation of the dependence of time-overthreshold upon the distance of closest approach to the anode wire $(y)$ for $5 \mathrm{GeV}$ pions. The points represent the mean value for each $y$ bin of width $200 \mu \mathrm{m}$, and the error bars represent the r.m.s. of the time-over-threshold distribution in each $y$ bin.

The time-over-threshold residual, $\Delta_{\mathrm{ToT}}$, is then calculated as a function of $y$ by subtracting the parameterised pion time-over-threshold $\left(\operatorname{ToT}_{\pi \mathrm{fit}}(y)\right)$ from the measured time-overthreshold $\left(\operatorname{ToT}_{\text {meas. }}(y)\right)$ :

$\Delta_{\mathrm{ToT}}=\operatorname{ToT}_{\text {meas. }}(y)-\operatorname{ToT}_{\pi \mathrm{fit}}(y)$.

This residual does not depend on the distance of closest approach (and therefore on the track length), as can be seen in Fig. 8. The values for pions are centred at zero, as expected, whereas those for electrons are shifted upwards on average by about $1.5 \mathrm{~ns}$. The corrected $\Delta_{\mathrm{ToT}}$ distributions for $5 \mathrm{GeV}$ pions (solid line) and electrons (dashed line) are shown in Fig. 9 for straws without highthreshold hits.

\subsubsection{Time-over-threshold studies with pseudo-tracks}

The energy loss measurements described above in detail for a single straw provide a powerful method for particle identification in gaseous detectors with many measurements available along the same track.

In the test beam, there was just one straw, providing a single time-over-threshold measurement per 


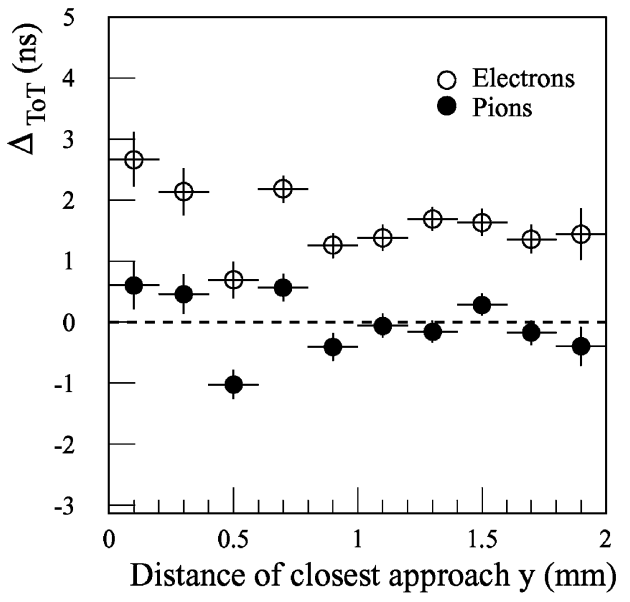

Fig. 8. Correlation between the corrected time-over-threshold residuals $\left(\Delta_{\mathrm{ToT}}\right)$ and the distance of closest approach for $5 \mathrm{GeV}$ pions and electrons. The points represent the mean value for each $y$ bin, and the error bars represent the statistical uncertainty on these mean values.

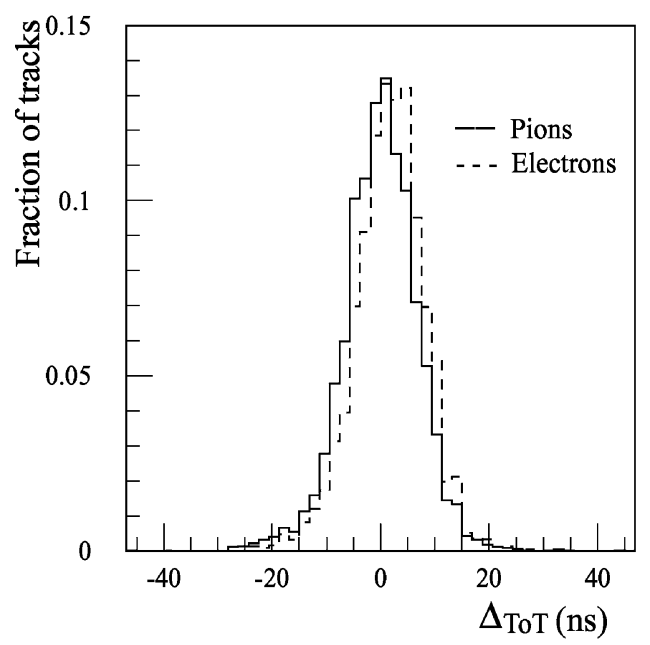

Fig. 9. Corrected time-over-threshold residuals, $\Delta_{\mathrm{ToT}}$, for a single straw without high-threshold hits and for samples of $5 \mathrm{GeV}$ pions and electrons (see text).

track. In the ATLAS TRT, there will be on average 35 straws crossed per track and a large fraction of those will give a hit (i.e. will yield a measured drift time within a two-standard-deviation window from the reconstructed position of the track). The hit efficiency depends partially on $\eta$ and largely on luminosity [9]. For the study shown

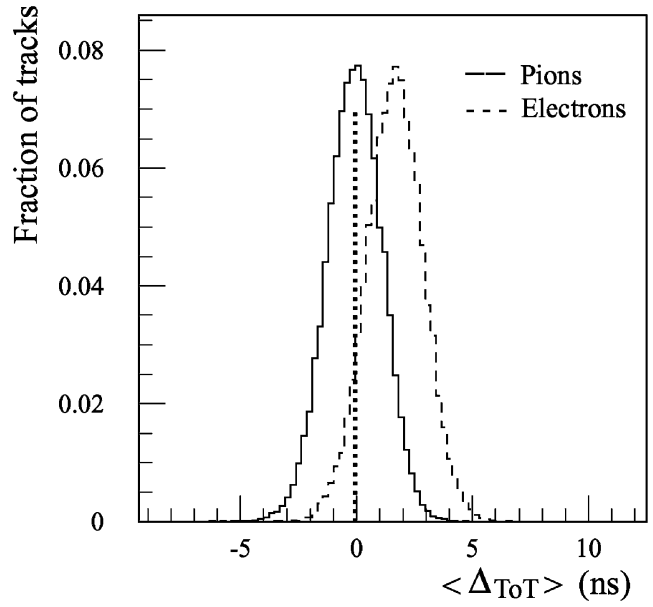

Fig. 10. $\left\langle\Delta_{\mathrm{ToT}}\right\rangle$ (no high-threshold hits) for $5 \mathrm{GeV}$ pseudotracks. The vertical line represents the $\left\langle\Delta_{\text {ToT }}\right\rangle$ value for a $90 \%$ electron efficiency.

in this section, which will be compared to ATLAS prediction for single tracks at $\eta=0.3$ (see Section 2.3.2), an hit efficiency of $90 \%$ is assumed. To simulate crudely the situation expected in the ATLAS experiment, pseudo-tracks were therefore created by randomly combining 32 hits (with a Gaussian spread of three hits) from different testbeam events. This analysis was performed with 30000 pseudo-tracks for each beam energy and particle type.

A discriminating variable, $\left\langle\Delta_{\mathrm{ToT}}\right\rangle$, is formed by averaging the time-over-threshold residuals from $n$ straws along the pseudo-track:

$\left\langle\Delta_{\mathrm{ToT}}\right\rangle=\frac{\sum_{i} \Delta_{\mathrm{ToT}}^{i}}{n}$ for $i=1, \ldots, n$.

Two $\left\langle\Delta_{\mathrm{ToT}}\right\rangle$ variables were studied: one used all hits available on the track; the other used only the hits without high-threshold discriminator level. These $\left\langle\Delta_{\mathrm{ToT}}\right\rangle$ distributions for pseudo-tracks are shown in Fig. 10 (no high-threshold hits) and Fig. 11 (all hits).

The distributions of the number of high-threshold hits along the reconstructed pseudo-tracks are shown in Fig. 12 for $5 \mathrm{GeV}$ pions and electrons. These distributions are the standard ones used for electron-pion separation using transition radiation. 


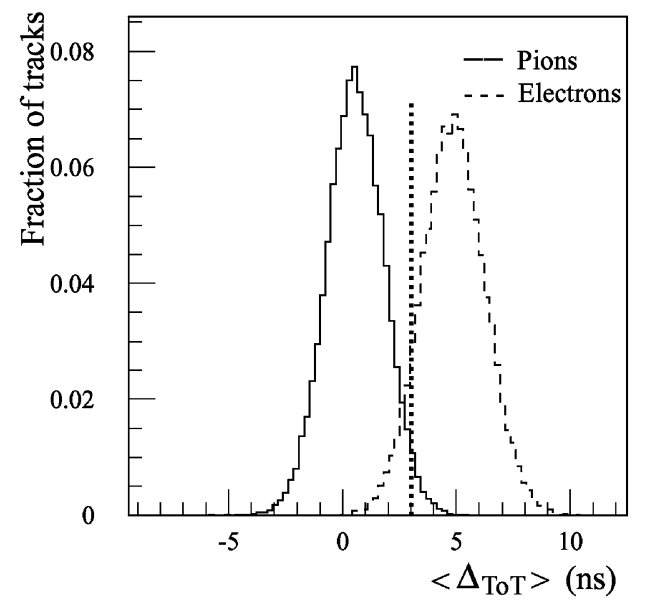

Fig. 11. $\left\langle\Delta_{\mathrm{ToT}}\right\rangle$ (all hits) for $5 \mathrm{GeV}$ pseudo-tracks. The vertical line represents the $\left\langle\Delta_{\mathrm{ToT}}\right\rangle$ value for a $90 \%$ electron efficiency.

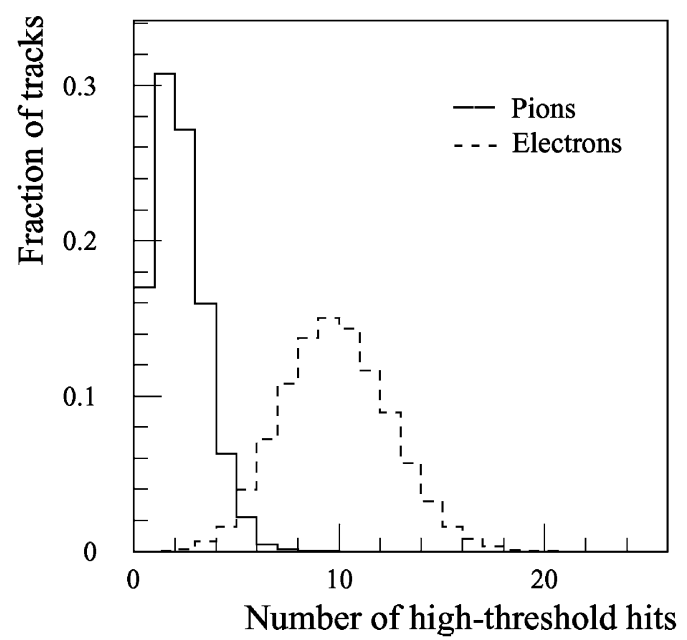

Fig. 12. Number of high-threshold hits for $5 \mathrm{GeV}$ pseudotracks.

\subsubsection{Performance obtained for electron-pion separation}

The way in which electron-pion separation can be achieved using the time-over-threshold method can be illustrated with the cut on the $\left\langle\Delta_{\mathrm{ToT}}\right\rangle$ variables shown in Figs. 10 (no high-threshold hits) and 11 (all hits). A $5 \mathrm{GeV}$ electrons could, in fact, be identified with $90 \%$ efficiency by requiring that $\left\langle\Delta_{\mathrm{ToT}}\right\rangle$ is above $3 \mathrm{~ns}$, when using all hits, or above $0 \mathrm{~ns}$, when excluding high-threshold hits.

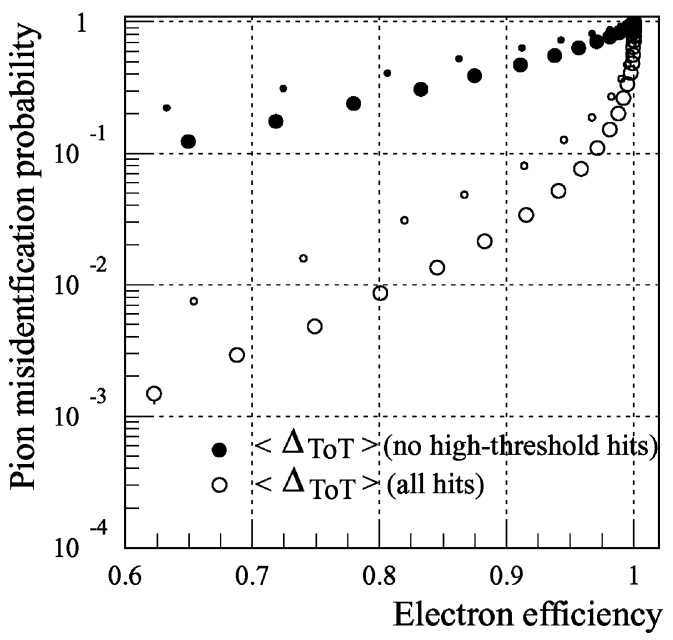

Fig. 13. Pion misidentification probability versus electron efficiency, as obtained using $\left\langle\Delta_{\mathrm{ToT}}\right\rangle$ at $5 \mathrm{GeV}$ for pseudotracks constructed from the test-beam data. The open circles are calculated using all the hits along the pseudo-track, whereas the black circles are calculated using only hits without highthreshold discriminator level. The large (small) circles correspond to $\left\langle\Delta_{\mathrm{ToT}}\right\rangle$ corrected (uncorrected) for the track position inside the straw.

The fraction of pions passing this cut represents the pion misidentification probability. This pion misidentification probability is shown in Fig. 13 as a function of the electron efficiency for the two $\left\langle\Delta_{\mathrm{ToT}}\right\rangle$ variables, namely using all hits (large open circles) and using only hits without high-threshold discriminator level (large black circles). When $\left\langle\Delta_{\mathrm{ToT}}\right\rangle$ is used as the only discriminating variable, much better electron-pion separation is obtained of course if all the hits on the track are used. In particular, a pion misidentification probability as low as $2.6 \%$ can be achieved for a $90 \%$ electron efficiency at $5 \mathrm{GeV}$. To illustrate the importance of the correction for the track position inside the straw (discussed in Section 2.2.1), the results without this correction (small circles) are also shown in Fig. 13. In the case where all hits are used, the improvement obtained by using this correction is about a factor 2.5 for an electron efficiency of $90 \%$.

In order to obtain the best possible electronpion separation, the time-over-threshold has to be combined with the cluster-counting technique, that 


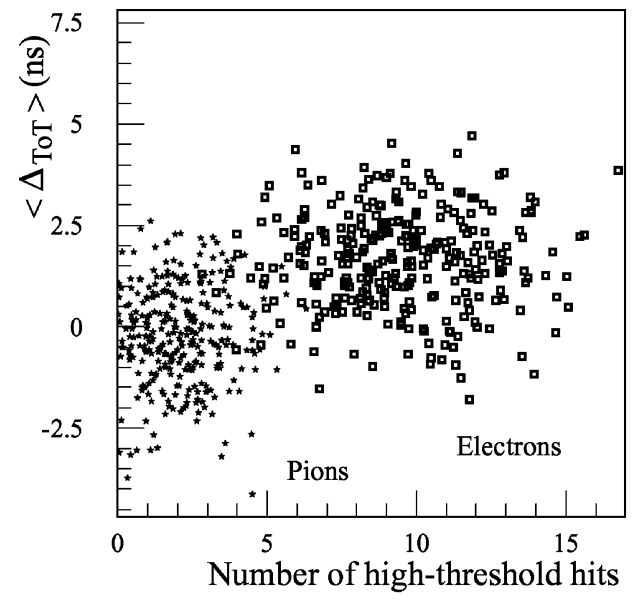

Fig. 14. Distribution of $\left\langle\Delta_{\mathrm{ToT}}\right\rangle$ (no high-threshold hits) versus number of high-threshold hits for $5 \mathrm{GeV}$ pseudo-tracks. Electrons are plotted as open squares and pions as black stars.

uses the number of high-threshold hits along the track. The correlation between $\left\langle\Delta_{\mathrm{ToT}}\right\rangle$ and the number of high-threshold hits is shown in Fig. 14 $\left(\left\langle\Delta_{\mathrm{ToT}}\right\rangle\right.$ without high-threshold hits) and Fig. 15 $\left(\left\langle\Delta_{\mathrm{ToT}}\right\rangle\right.$ with all hits) for $5 \mathrm{GeV}$ electrons and pions. While there is now one more degree of freedom for optimising electron identification, the two discriminating variables are correlated. The correlation is clearly stronger, as expected, when $\Delta_{\text {ToT }}$ is averaged over all the hits on the pseudotrack. A likelihood analysis has been performed to combine the two variables in an optimal way for three different test-beam energies of 5, 10 and $20 \mathrm{GeV}$. The likelihood analysis gives very similar results for the combination of the number of high-threshold hits with any of the two time-overthreshold variables. The combined method mentioned in this article refers at the combination when all hits are used for the time-over-threshold variable. In Table 1 , the pion misidentification probability at $90 \%$ electron efficiency for various time-over-threshold methods, for the clustercounting technique and for the combined method is tabulated as a function of energy. The comparison between the electron-pion separation achieved at $5 \mathrm{GeV}$ with the cluster-counting technique and the combined method is shown in Fig. 16, which can also be compared to Fig. 13,

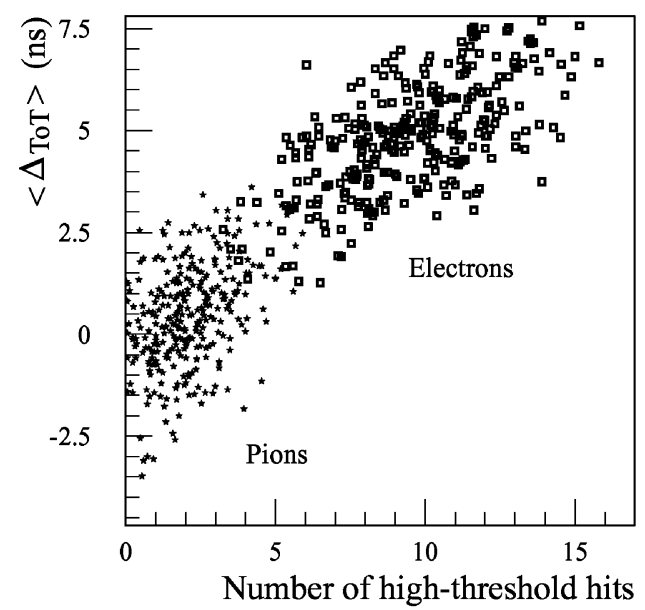

Fig. 15. Distribution of $\left\langle\Delta_{\mathrm{ToT}}\right\rangle$ (all hits) versus number of high-threshold hits for $5 \mathrm{GeV}$ pseudo-tracks. Electrons are plotted as open squares and pions as black stars.

where only the time-over-threshold information is used. Table 1 clearly demonstrates that the transition-radiation information provides a rejection which is a factor 3.5-6 higher than that obtained with time-over-threshold alone. Nevertheless, the pion misidentification probability at $5 \mathrm{GeV}$ and at $90 \%$ electron efficiency improves by a factor of two for the combined method with respect to the standard transition-radiation cluster-counting technique alone. No improvement is seen at $20 \mathrm{GeV}$. This is not surprising, since the performance of particle identification techniques based on the energy loss degrades with increasing energy.

\subsection{Monte Carlo simulation and comparison to test-beam data}

\subsubsection{Monte Carlo model}

A Monte Carlo model, based on GEANT 3.21 [10], has been developed to simulate the straws and the other detectors in the beam line [11]. It includes detailed models to describe the energy deposition in the straws, transition-radiation creation and absorption and the response of the front-end electronics [12]. The energy loss for each charged particle crossing the ionisation gas is calculated using the Photo-Absorption Ionisation (PAI) 
Table 1

Pion misidentification probability obtained using different techniques (see text), for a $90 \%$ electron efficiency and for pseudo-tracks constructed from test-beam data at three different energies

\begin{tabular}{lccc}
\hline Method & $E_{\mathrm{b}}=5 \mathrm{GeV}$ & $E_{\mathrm{b}}=10 \mathrm{GeV}$ & $E_{\mathrm{b}}=20 \mathrm{GeV}$ \\
\hline $\begin{array}{l}\text { Time-over-threshold } \\
\text { (no high-threshold hits) }\end{array}$ & $0.46 \pm 0.01$ & $0.58 \pm 0.01$ & $0.73 \pm 0.01$ \\
Time-over-threshold & & & $0.10 \pm 0.01$ \\
(all hits, no $y$ correction) & $0.067 \pm 0.002$ & $0.041 \pm 0.001$ & $0.17 \pm 0.01$ \\
Time-over-threshold (all hits) & $0.026 \pm 0.001$ & $0.012 \pm 0.001$ & $0.10 \pm 0.002$ \\
Number of high-threshold hits & $0.0072 \pm 0.0003$ & $0.008 \pm 0.001$ & $0.017 \pm 0.001$ \\
Combined method & $0.0037 \pm 0.0002$ & $0.016 \pm 0.001$ \\
\hline
\end{tabular}

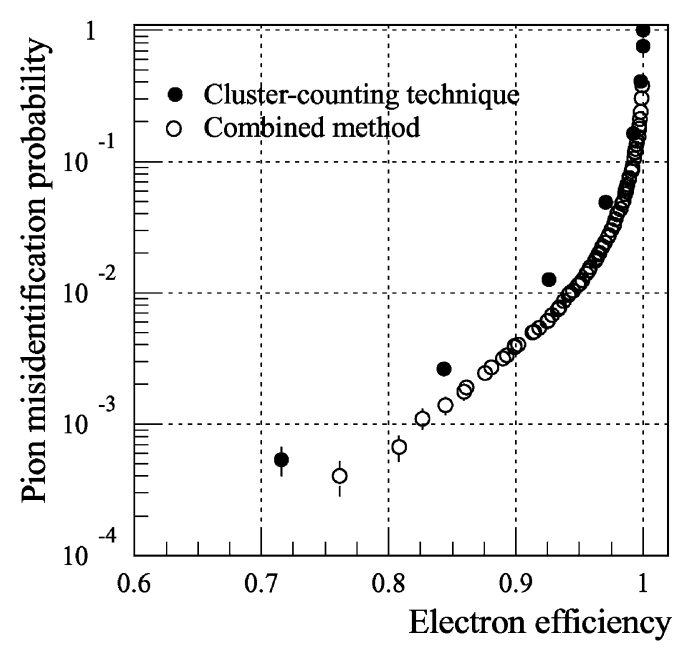

Fig. 16. Pion misidentification probability versus electron efficiency at $5 \mathrm{GeV}$ for pseudo-tracks constructed from testbeam data. The results are shown for the standard clustercounting technique and for the combined method using also the time-over-threshold information (see text and Table 1).

model [13]. This ionisation is deposited over a small (typically $\sim 50$ per $\mathrm{cm}$ for minimumionising particles, while $\sim 70$ per $\mathrm{cm}$ for particles at the Fermi plateau) number of primary ionisation centres along the path length. In addition, for a charged particle with a Lorentz factor above 1000, transition-radiation photons are generated in the radiator material, according to the formulae given in Refs. [14,15]. This photon spectrum is transported along the particle track, partially absorbed in the radiator itself, in the straw walls, and in the gas mixture inside the straws. Transition-radiation photons deposit point-like ionisa- tion clusters in the same straws as those crossed by the parent particle. All the primary clusters are then allowed to drift towards the anode, as shown in Fig. 2, and the signal amplitudes and arrival times on the wire are summed over all primary clusters for all charged particles crossing each straw. Space-charge effects, that cause a reduction in the gain for large energy depositions are also simulated. This summed signal is then folded with a model of the electronics signal shape as shown in Fig. 17. The resulting signal is finally discriminated and digitised in $3.125 \mathrm{~ns}$ bins.

In order to compare the PAI model with experimental data in detail for the ionisation losses of pions and electrons, the pulse-height information from the straw prototype (for the configuration without radiators) placed between the two silicon detectors was used. The comparison between the Monte Carlo and experimental differential energy spectra is shown in Figs. 18 and 19 for $5 \mathrm{GeV}$ pions and electrons, respectively. The most probable energies are $1.1 \mathrm{keV}$ for pions and $1.3 \mathrm{keV}$ for electrons.

The integral energy deposition spectra for $5 \mathrm{GeV}$ pions and electrons and for data and Monte Carlo are shown in Fig. 20. The integral spectra are obtained from the differential spectra above the low threshold of $0.2 \mathrm{keV}$, chosen for the operation of the ATLAS TRT front-end electronics.

The good agreement observed between the Monte Carlo and the experimental data for the differential and integral spectra above $0.2 \mathrm{keV}$ is an essential ingredient in the work required to obtain reliable predictions for the electron-pion 

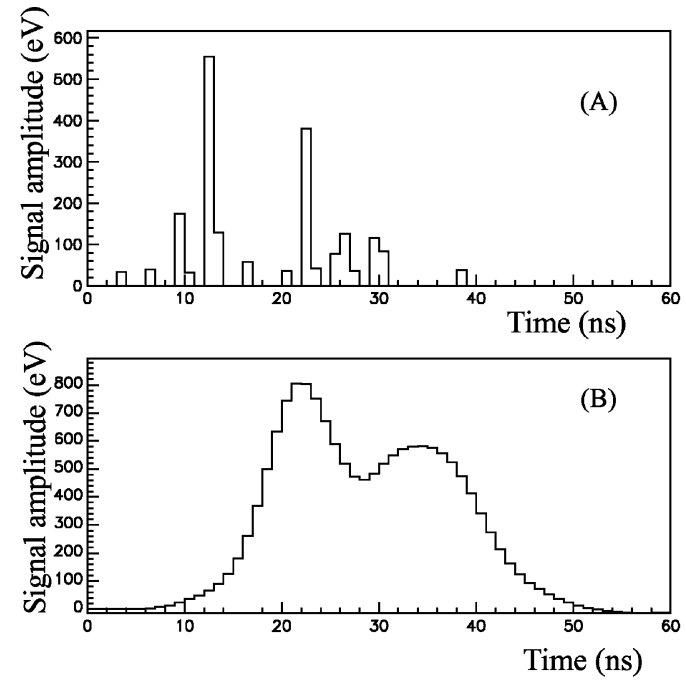

Fig. 17. Monte Carlo simulation of the straw signal. (A) The ionisation clusters drift towards the anode and the signal amplitudes and arrival times are recorded. (B) The straw output signal is then convoluted with a model of the response of the front-end electronics.

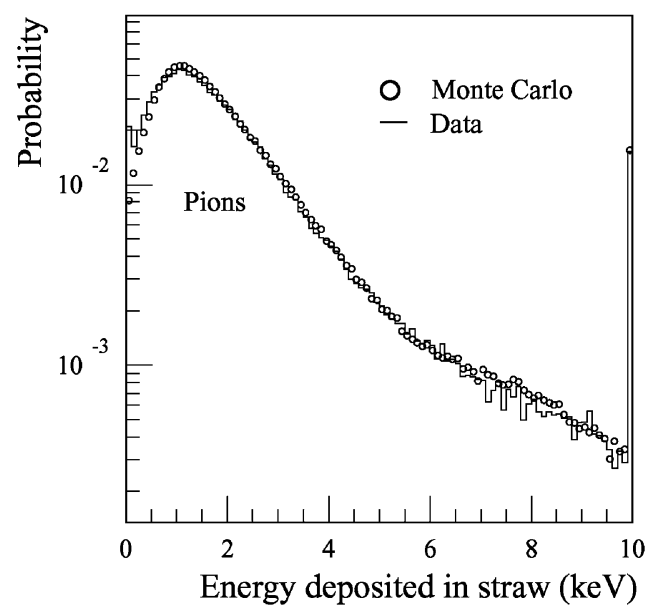

Fig. 18. Differential energy spectra from test-beam data (histograms) and Monte Carlo simulation (open circles) for a single straw without radiator and for $5 \mathrm{GeV}$ pions.

separation to be expected in ATLAS using the methods described above. For both the Monte Carlo and the data time-over-threshold analysis, the same software chain has been used. The pion misidentification probability at $90 \%$ electron

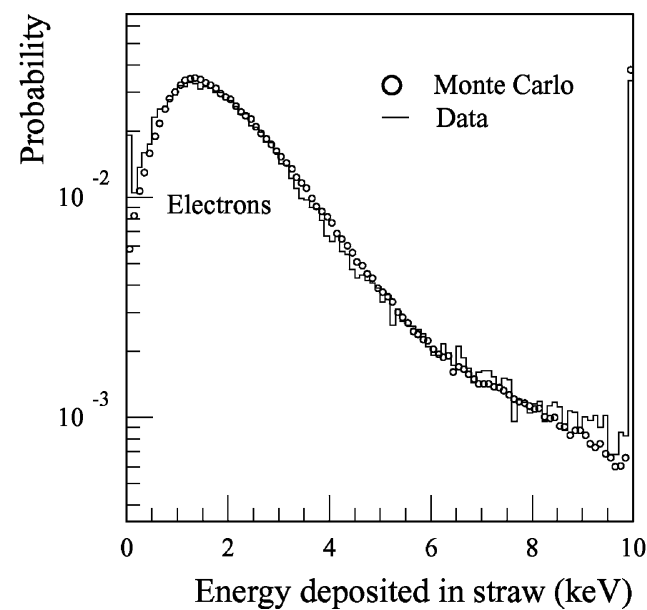

Fig. 19. Differential energy spectra from test-beam data (histograms) and Monte Carlo simulation (open circles) for a single straw without radiator and for $5 \mathrm{GeV}$ electrons.

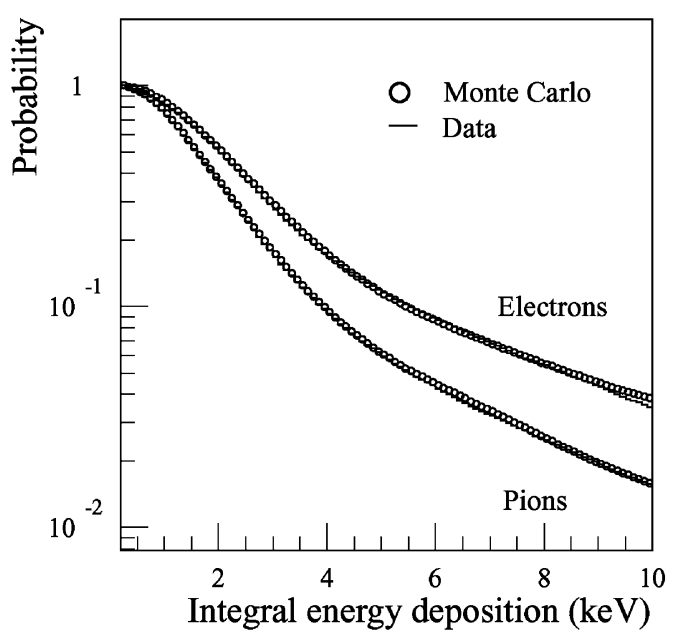

Fig. 20. Integral energy deposition spectra (integrated above $0.2 \mathrm{keV}$ ) for $5 \mathrm{GeV}$ pions and electrons, as measured in the testbeam and predicted by Monte Carlo simulations for a single straw without radiator. The Monte Carlo clearly reproduces well the data.

efficiency is shown in Fig. 21 as a function of energy for the test-beam data at 5, 10 and $20 \mathrm{GeV}$ and for the Monte Carlo simulation. The results are shown for pseudo-tracks and for the various methods described above. Additional Monte Carlo simulation results at lower energies (1 and $2 \mathrm{GeV}$ ) are also shown in Fig. 21. The four sets of points 


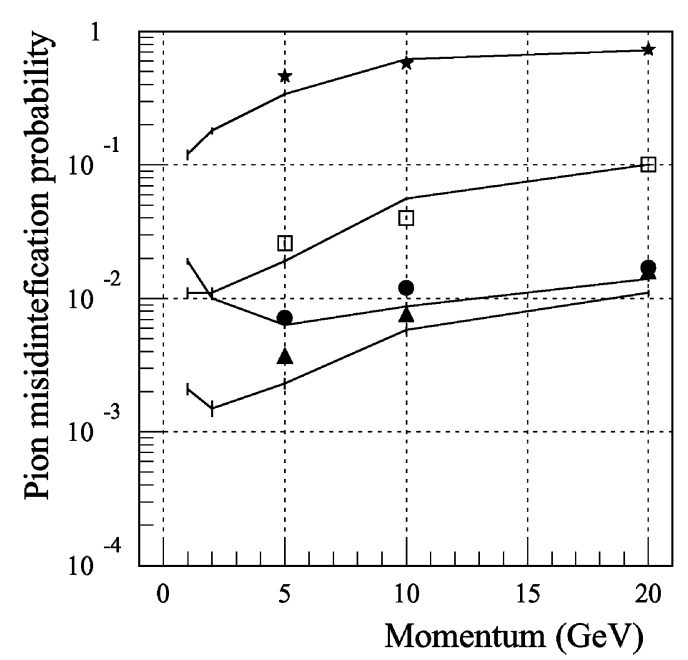

Fig. 21. Pion misidentification probability as a function of momentum at $90 \%$ electron efficiency for pseudo-tracks in the test-beam setup. The performance is shown for $\left\langle\Delta_{\mathrm{ToT}}\right\rangle$, without using high-threshold hits (stars), for $\left\langle\Delta_{\mathrm{ToT}}\right\rangle$ using all hits (squares), for the transition-radiation cluster-counting technique (circles) and for the overall combined method (triangles). The test-beam data results at 5,10 and $20 \mathrm{GeV}$ are shown as points, while the Monte Carlo simulation results are shown as lines from 1 to $20 \mathrm{GeV}$. The errors shown are statistical (above $5 \mathrm{GeV}$ the errors are often smaller than the symbols).

(data) and lines (Monte Carlo) represent (in order of decreasing pion misidentification probability) the time-over-threshold method alone (without using high-threshold hits and using all hits), the cluster-counting technique alone and the overall combined method. In the lower-energy region (1-2 GeV), where transition radiation production is not yet saturated, the time-over-threshold method is expected to improve the overall electron-identification performance by up to one order of magnitude.

\subsubsection{Expected performance for $\mathrm{e} / \pi$ separation in the ATLAS TRT}

The ATLAS Inner Detector is described in detail in Ref. [1]. It combines high-resolution silicon detectors (using both pixel and micro-strip technologies) at the inner radii with the 'continuous' tracking elements of the TRT at the outer radii, all contained in a $2 \mathrm{~T}$ solenoidal magnetic field.
The electron-pion separation was studied in more detail for the ATLAS TRT by using the full simulation (based on GEANT 3.21 and the PAI model) of single particles without pile-up and with the overall straw response tuned to test-beam data. Tracks were generated in the barrel TRT, in a narrow region around $\eta=0.3(0.29<\eta<0.31)$ and at different momenta. This $\eta$ region corresponds approximately to the incidence angle of $21^{\circ}$ of the beam tracks with respect to the straw axis in the test-beam setup, and gives on average 32 hits per track (see Section 2.2.2).

These detailed studies are necessary to validate the time-over-threshold predictions reported in Section 2.3.1, due to the somewhat different radiator geometry in the test-beam setup and since pseudo-tracks do not take into account possible correlations between successive straws on a track. Such correlations are expected to be energydependent to some extent and are due, in particular, to hadronic interactions, electromagnetic showers, high-energy $\delta$-rays and transition radiation. The reconstruction of the tracks was performed using a standard ATLAS pattern recognition code, which starts by associating hits in the TRT and then projects the track candidates back to the silicon hits [16].

The resulting pion misidentification probability is shown in Fig. 22 as a function of $p_{\mathrm{T}}$, for a $90 \%$ electron efficiency and for the different methods discussed above.

For a transverse momentum of $5 \mathrm{GeV}$, the timeover-threshold method improves the overall TRT performance by a factor of two, while at even lower energies of $1-2 \mathrm{GeV}$ the improvement is expected to be as large as one order of magnitude, in agreement with the test-beam data pseudo-track predictions discussed in Section 2.3.1.

\subsubsection{Comparison between pseudo-tracks and the full ATLAS Monte Carlo}

The performance predicted for the electronpion separation with the full ATLAS simulation (see Fig. 22) is consistent with the studies done with pseudo-tracks obtained from test-beam data (see Fig. 21) for the time-over-threshold method without using high-threshold hits. However, the predicted performance of the time-over-threshold 
method with all hits, of the cluster-counting technique and of the combined electron-pion separation are significantly worse in the case of the ATLAS barrel TRT Monte Carlo.

In order to separate out the effect due to transition radiation from other effects, such as

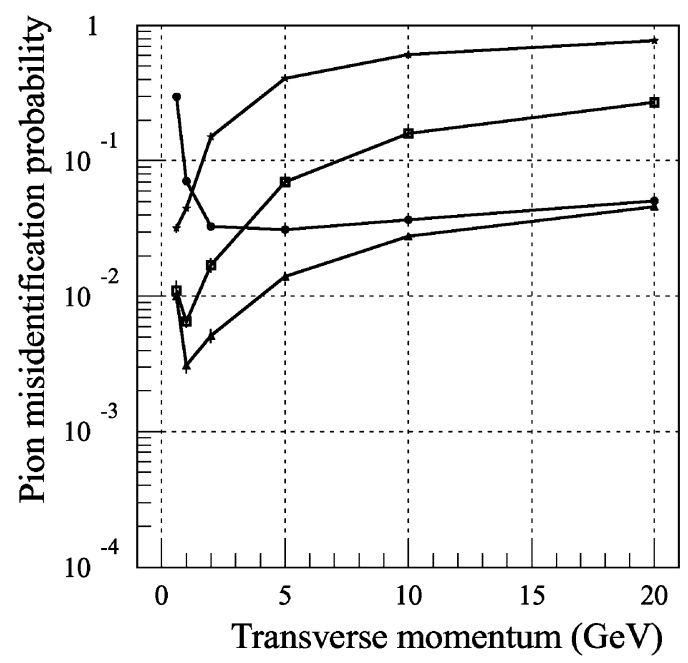

Fig. 22. Pion misidentification probability as a function of transverse momentum at $90 \%$ electron efficiency for simulated single tracks at $\eta=0.3$ in the ATLAS Inner Detector without pile-up. The performance is shown for $\left\langle\Delta_{\mathrm{ToT}}\right\rangle$ without using high-threshold hits (stars), for $\left\langle\Delta_{\mathrm{ToT}}\right\rangle$ using all hits (squares), for the transition-radiation cluster-counting technique (circles), and for the overall combined method (triangles). possible correlations between successive straws along a track (neglected obviously in the pseudotrack analysis described in Section 2.2), the radiator model used for the full ATLAS barrel TRT Monte Carlo was tuned to roughly reproduce the test-beam transition-radiation yields. As a result of this tuning, the radiator yield in the ATLAS barrel TRT Monte Carlo had to be increased by $20 \%$ to account for the differences between the radiator used in the test-beam and the radiator planned to be used for the barrel TRT. Tracks were then generated in the barrel TRT and compared to test-beam Monte Carlo pseudotracks, that have been shown in Section 2.3.1 to reproduce reliably the test-beam data. The resulting pion misidentification probabilities at $90 \%$ electron efficiency are tabulated in Table 2, for the transition-radiation cluster-counting technique, the time-over-threshold method without highthreshold hits and the time-over-threshold method with all hits. The results are shown as a function of momentum for fully simulated tracks and for testbeam pseudo-tracks.

The results now show a good agreement between the predictions from barrel TRT tracks with tuned radiator performance and from testbeam pseudo-tracks, in the case of the standard transition-radiation cluster-counting technique. The discrepancies observed between test-beam pseudo-tracks and barrel TRT tracks, in the case

Table 2

Pion-misidentification probability obtained using different techniques (see text), for a $90 \%$ electron efficiency and for barrel TRT tracks and test-beam pseudo-tracks at different momenta. The barrel TRT tracks were simulated at $\eta=0.3$, corresponding to an incident angle close to the test-beam one. The performance obtained with the standard transition-radiation cluster-counting technique are tabulated in the first block, while the performance obtained with the time-over-threshold method without high-threshold hits (with all hits) are tabulated in the second (third) block

\begin{tabular}{lccc}
\hline Monte Carlo tracks type & $p=5 \mathrm{GeV}$ & $p=10 \mathrm{GeV}$ & $p=20 \mathrm{GeV}$ \\
\hline TR technique & & & $0.012 \pm 0.001$ \\
Barrel TRT tracks & $0.007 \pm 0.001$ & $0.008 \pm 0.001$ & $0.014 \pm 0.001$ \\
Test-beam pseudo-tracks & $0.006 \pm 0.001$ & $0.009 \pm 0.001$ & $0.77 \pm 0.01$ \\
ToT method (no HT hits) & & & $0.72 \pm 0.01$ \\
Barrel TRT tracks & $0.41 \pm 0.01$ & $0.61 \pm 0.01$ & $0.62 \pm 0.01$ \\
Test-beam pseudo-tracks & $0.34 \pm 0.01$ & & $0.14 \pm 0.01$ \\
ToT method (all hits) & & $0.075 \pm 0.001$ & $0.10 \pm 0.01$ \\
Barrel TRT tracks & $0.031 \pm 0.001$ & $0.056 \pm 0.001$ & \\
Test-beam pseudo-tracks & $0.019 \pm 0.001$ & & \\
\hline
\end{tabular}


of the time-over-threshold methods, are significant (up to $\sim 50 \%$ in terms of pion misidentification probability) and are most likely due to the correlation effects mentioned above.

\section{Expected performance for $K / \pi$ separation in the ATLAS TRT}

Hadron identification is obviously important for many aspects of B-physics studies, in the ATLAS experiment [17]. Earlier Monte Carlo studies of $\mathrm{K} / \pi$ separation using signal shape information from the ATLAS TRT are reported in Ref. [6]. In that work, only the expected shift in the time of the leading edge as a function of particle mass was used, and a maximum $\mathrm{K} / \pi$ separation of 0.9 standard deviation was predicted at $5 \mathrm{GeV}$ momentum.

In this paper, the time-over-threshold method is used, assuming that the TRT readout will provide this information at low luminosity while preserving the output bandwidth requirements. The analysis of the time-over-threshold follows the same procedure as described above and, obviously, all hits are used in this case. The expected $\left\langle\Delta_{\mathrm{ToT}}\right\rangle$ distributions for reconstructed tracks in the ATLAS barrel TRT at $|\eta|=0.3$ are shown in Fig. 23 for $5 \mathrm{GeV}$ pions and kaons. The expected $\mathrm{K} / \pi$ separation as a function of transverse momentum is shown in Fig. 24 in units of standard deviation. Without including any pile-up effects, the $\mathrm{K} / \pi$ separation is predicted to be above one standard deviation for transverse momenta between 2 and $5 \mathrm{GeV}$, averaged over the full rapidity coverage (solid line), and above one standard deviation over a broader $p_{\mathrm{T}}$-range between 2 and $15 \mathrm{GeV}$ at $|\eta|=0.3$ (dashed line).

As an example of the importance of having some $\mathrm{K} / \pi$ separation (and, more generally, hadron identification) in ATLAS, the results of this study have been applied to the $\mathrm{B}_{\mathrm{d}}^{0} \rightarrow \pi^{+} \pi^{-}$ decay, which is useful for extracting information about the angle $\alpha$ of the unitarity triangle used for CP-violation studies. The two-pion final state of the $\mathrm{B}_{\mathrm{d}}^{0}$ decay is plagued by various sources of background, mostly three- or two-body decays involving also kaons and protons. The predicted

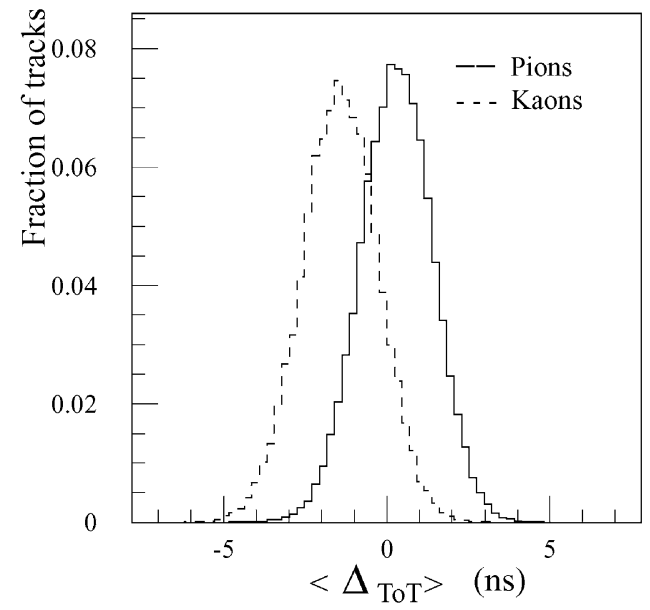

Fig. 23. $\left\langle\Delta_{\mathrm{ToT}}\right\rangle$ (all hits) for reconstructed tracks in the ATLAS barrel TRT at $|\eta|=0.3$ for $5 \mathrm{GeV}$ pions (solid line) and kaons (dashed line).

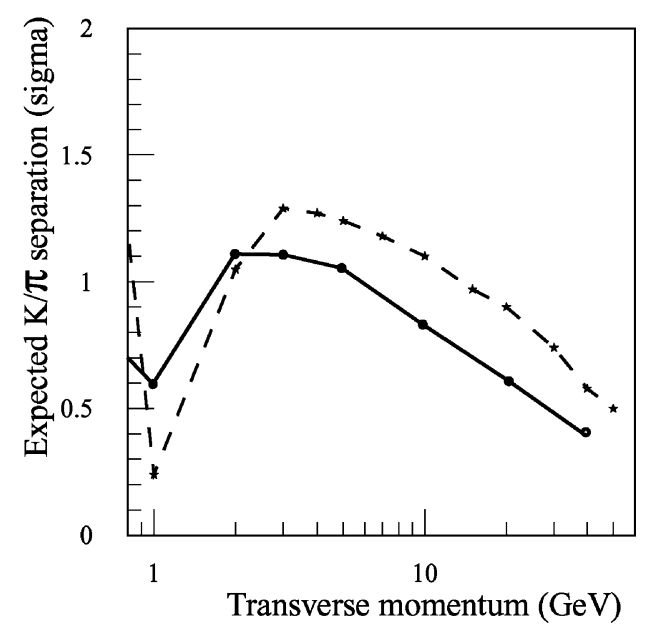

Fig. 24. Expected $K / \pi$ separation in the ATLAS TRT as a function of transverse momentum (no pile-up effects included). The separation is shown as an average over the full rapidity coverage (solid line) and at $|\eta|=0.3$ (dashed line).

$\pi^{+} \pi^{-}$invariant mass spectrum for the ATLAS experiment, after 3 years of low-luminosity datataking at the LHC is shown in Fig. 25, using recent values of the $\mathrm{B}_{\mathrm{d}}^{0}$ branching ratios (as quoted in Ref. [18]), and for a scenario without hadron identification in the ATLAS TRT. The signal is represented by the dark shaded area, while the 


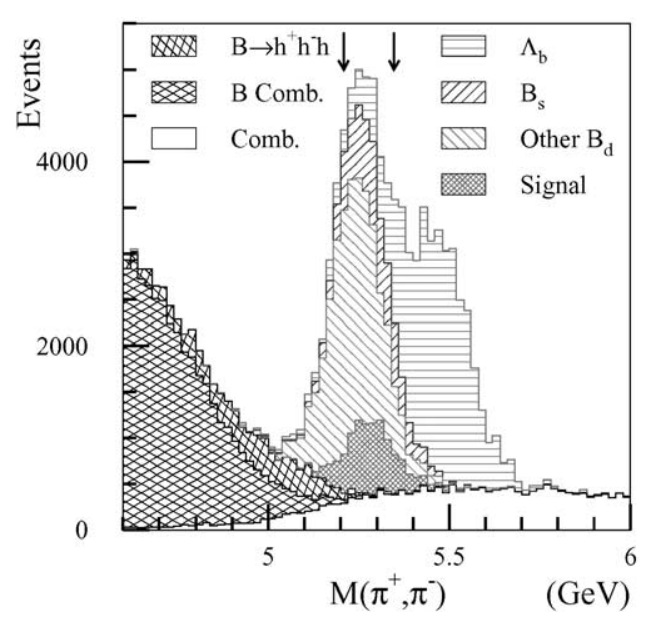

Fig. 25. $\pi^{+} \pi^{-}$mass spectrum in the ATLAS experiment for $B_{d}^{0}$ signal and various backgrounds and for the scenario without kaon-pion separation in the ATLAS TRT.

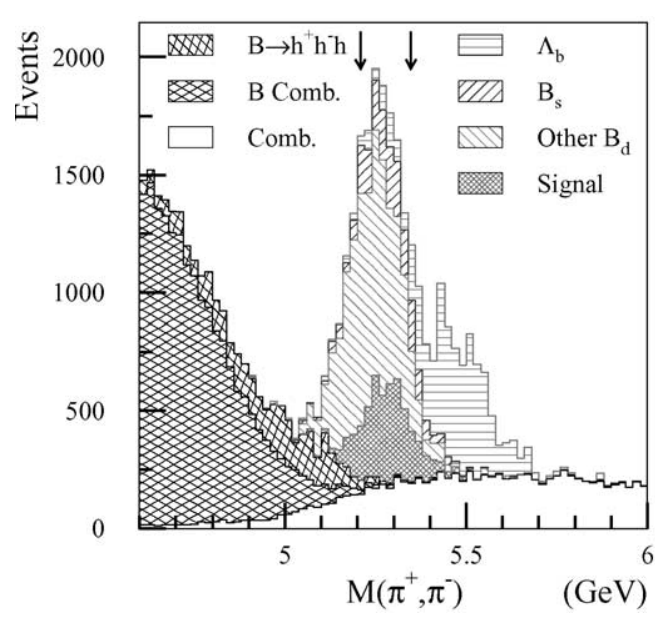

Fig. 26. $\pi^{+} \pi^{-}$mass spectrum in the ATLAS experiment for $\mathrm{B}_{\mathrm{d}}^{0}$ signal and various backgrounds and for the scenario with a kaon-pion separation in the ATLAS TRT as predicted by the study presented in this paper (see text).

various backgrounds (most of which lie just on top of the signal) are represented as lighter shaded regions [18]. As an illustration, the corresponding predicted $\pi^{+} \pi^{-}$invariant mass spectrum when $\mathrm{d} E /$ $\mathrm{d} x$ information (via the time-over-threshold measurements in the TRT) is used is shown in Fig. 26, for a $50 \%$ efficiency for the $\mathrm{B}_{\mathrm{d}}^{0} \rightarrow \pi^{+} \pi^{-}$signal. The fraction of the signal in a window around the $\mathrm{B}_{\mathrm{d}}^{0}$ mass improves from a value of $15 \%$ to a value of
$21 \%$. The accuracy of the measurement of the $\mathrm{B}_{\mathrm{d}}^{0}$ $\rightarrow \pi^{+} \pi^{-}$asymmetry, which makes use of $\mathrm{d} E / \mathrm{d} x$ information together with other information in an event-by-event maximum likelihood, is improved by about $35 \%$ with respect to the scenario without hadron identification in the ATLAS Inner Detector.

\section{Conclusions}

Using test-beam data taken at the CERN SPS with 5, 10 and $20 \mathrm{GeV}$ pion and electron beams, time-over-threshold measurements, using the front-end electronics planned for the operation of the ATLAS TRT at the LHC, have demonstrated that significant particle identification can be achieved over an interesting range of particle types and energies.

Electron-pion separation at energies below $10 \mathrm{GeV}$ can be significantly enhanced by combining the standard transition-radiation clustercounting technique with the time-over-threshold information. In particular, pion misidentification probabilities below $4 \times 10^{-3}$ can be reached at $90 \%$ electron efficiency for energies between 1 and $5 \mathrm{GeV}$. This measured test-beam performance leads to expected pion misidentification probability of about $10^{-2}$ at $90 \%$ electron efficiency over the same energy range in the ATLAS barrel TRT. Detailed Monte Carlo studies are shown to reliably reproduce the test-beam results.

The extrapolation to $\mathrm{K} / \pi$ separation in the field of B-physics at the LHC leads to the conclusion that the ATLAS B-physics potential could be improved through the use of time-over-threshold measurements in the ATLAS TRT.

\section{Acknowledgements}

Work was partially supported by grant no. $620 /$ E-77/SPUB/CERN/P-03/DZ $2 / 9$ of the Polish Committee for Scientific Research (KBN).

\section{References}

[1] ATLAS Inner Detector Technical Design Report, CERN/LHCC/97-17, ATLAS TDR 5, 1997. 
[2] J.T. Shank, et al., Nucl. Instr. and Meth. A 310 (1991) 133.

[3] T. Akesson, et al., Nucl. Instr. and Meth. A 372 (1996) 70.

[4] T. Akesson, et al., Nucl. Instr. and Meth. A 412 (1998) 200.

[5] V. Bashkirov, B. Dolgoshein, ATLAS INDET-No-090, 1994.

[6] ATLAS Detector and Physics Performance Technical Design Report, CERN/LHCC/99-14,15, ATLAS TDR 14, 1999, pp. 74-76 and ATLAS TDR 15, 1999, pp. 577582.

[7] V. Bashkirov, Nucl. Instr. and Meth. A 433 (1999) 560.

[8] T. Akesson, et al., Nucl. Instr. and Meth. A 449 (2000) 446.

[9] ATLAS Inner Detector Technical Design Report, CERN/LHCC/97-16, ATLAS TDR 4, 1997, pp. 95-96.

[10] CN division Application Software group, GEANT detector description and simulation tool, CERN Program Library Long Writeup W5013, 1993.
[11] A. Artamonov, et al., "DICE-95", internal note ATLASSOFT/95-14, CERN, 1995.

[12] ATLAS Inner Detector Technical Design Report, CERN/LHCC/97-16, ATLAS TDR 4, 1997, pp. 90-91.

[13] V.M. Grishin, V.K. Ermilova, S.K. Kotelnikov, Nucl. Instr. and Meth. A 307 (1991) 273.

[14] M. Cherry, G. Hartmann, D. Muller, T.A. Prince, Phys. Rev. 10 (1974) 3594.

[15] G.M. Garibian, L.A. Gevorgian, C. Yang, Nucl. Instr. and Meth. 125 (1975) 133.

[16] I. Gavrilenko, ATLAS Internal Note, ATLAS-INDETNo-165, 1997.

[17] ATLAS Detector and Physics Performance Technical Design Report, CERN/LHCC/99-15, ATLAS TDR 15, 1999, pp. 561-618.

[18] G. Altarelli, M.L. Mangano (Eds.), Proceedings of the workshop on standard model physics (and more) at the LHC, CERN 2000-004, pp. 329-333. 